\title{
Sjögren's syndrome autoantibodies provoke changes in gene expression profiles of inflammatory cytokines triggering a pathway involving TACE/NF- $\kappa \mathrm{B}$
}

\author{
Sabrina Lisi ${ }^{1,4}$, Margherita Sisto ${ }^{1,4}$, Dario Domenico Lofrumento² and Massimo D'Amore ${ }^{3}$
}

We explore the association of the inflammatory gene expression profile observed in the chronic inflammatory autoimmune disorder Sjögren's syndrome (SS) with changes in TNF- $\alpha$ converting enzyme (TACE), tumor necrosis factor (TNF)- $\alpha$ and nuclear factor (NF)- $\kappa$ B levels showing that pathways that include TNF- $\alpha$ signaling converge on NF- $\kappa$ B contributing to exacerbate the diseases. The treatment of human salivary gland epithelial cells (SGECs) with SS anti-Ro/SSA autoantibodies (Abs) result in a progressive increase in NF- $\kappa$ B-DNA binding, that includes a marked enhancement in NF- $\kappa B$ subunit p65 protein-DNA binding. A human cytokine multi-analyte array demonstrated that the NF- $\kappa$ B proinflammatory target genes, increased by anti-Ro/SSA Abs treatment, includes CXC chemokines (CXCL1, CXCL6 and CXCL9), CC chemokines (CCL2, CCL13 and CCL20), interleukins (IL-1 $\alpha$, IL-1 $\beta$, IL-1F8, IL-6, IL-8, IL-9, IL-13, IL-17 and IL-22) and their receptors (IL-1RN, IL-10R $\alpha$, IL-13R $\alpha$, CCR1, CCR2, CCR3, CCR4 and CXCR1). Blockade of TACE through the use of the specific inhibitor TAPI-1 regulates proinflammatory cytokines production in SGEC treated with anti-Ro/SSA Abs inhibiting $\mathrm{NF}-\kappa \mathrm{B}$ nuclear translocation and activation. To further investigate the role of NF- $\kappa \mathrm{B}$ on anti-Ro/SSA Abs-determined proinflammatory gene expression, we used the inhibitory protein $1 \kappa \mathrm{B}-\alpha$ dominant negative super-repressor as inhibitor of NF- $\kappa$ B-DNA binding, demonstrating that transfection with dominant-negative $1 \kappa \mathrm{B}-\alpha$ in anti-Ro/SSA-treated SGEC determined a marked reduction of proinflammatory cytokines gene expression. Although further studies are needed to clarify the mechanisms underlying SS, our results demonstrate that SS Abs exert their pathogenic effects via triggering the TACE/TNF- $\alpha /$ NF- $\kappa$ B axis.

Laboratory Investigation (2012) 92, 615-624; doi:10.1038/labinvest.2011.190; published online 12 December 2011

KEYWORDS: anti-Ro/SSA autoantibodies; I $\kappa \mathrm{B}-\alpha$; NF- $\kappa$ B; Sjögren's syndrome; TACE; TNF- $\alpha$

Nuclear factor- $\kappa \mathrm{B}(\mathrm{NF}-\kappa \mathrm{B})$ transcription is increased by multiple mechanisms that promote additional proinflammatory gene induction through further increasing $\mathrm{NF}-\kappa \mathrm{B}$ transcription. ${ }^{1}$ This positive progressive gene induction is implicated in autoimmunity. ${ }^{2} \mathrm{NF}-\kappa \mathrm{B}$ is activated by various intracellular signals, including tumor necrosis factor (TNF) $-\alpha$. TNF- $\alpha$ has been found in functionally and structurally damaged areas of the salivary glands and has been implicated in the disease pathogenesis of Sjögren's syndrome (SS). ${ }^{3,4}$ Sjögren's syndrome is a systemic chronic inflammatory autoimmune disorder that affects secretory organs such as the salivary and lachrymal glands, ${ }^{5}$ and TNF- $\alpha$ production seems to be closely linked to SS. ${ }^{4,6,7}$ In addition, proteases such as the TNF- $\alpha$ converting enzyme (TACE) contribute to the autoimmune condition by processing innate inflammatory cytokines and other proteins. ${ }^{8}$ Cytokines have a central role in triggering and perpetuating inflammation in the secretory glands, and the imbalance of pro-over anti-inflammatory cytokines results in cumulative damage to the glands impairing the secretory function. ${ }^{8}$ It is apparent from previous literature and work carried out in our laboratory that TNF- $\alpha$ is rapidly produced in the SS salivary gland in response to tissue injury. ${ }^{8-10}$ This cytokine is released in soluble form from its membrane-bound precursor by the membrane-anchored zinc metalloproteinase TACE. ${ }^{11,12}$ In human salivary gland epithelial cells (SGECs)

\footnotetext{
${ }^{1}$ Department of Human Anatomy and Histology, Laboratory of Cell Biology, University of Bari Medical School, Bari, Italy; ${ }^{2}$ Department of Biological and Environmental Sciences and Technologies, University of Salento, Lecce, Italy and ${ }^{3}$ Department of Internal Medicine and Public Medicine, Section of Rheumatology, University of Bari Medical School, Bari, Italy

Correspondence: Dr S Lisi, Department of Human Anatomy and Histology, University of Bari, Piazza Giulio Cesare 1, I-70124 Bari, Italy. E-mail: s.lisi@anatomia.uniba.it

${ }^{4}$ These authors contributed equally to this work.

Received 4 July 2011; revised 4 October 2011; accepted 11 October 2011
} 
treated with the anti-Ro/SSA autoantibodies (Abs) characterizing SS, the increase in soluble TNF- $\alpha$ levels is preceded by an increase in TACE activity. ${ }^{13}$ In addition, through shedding and activation of TACE, anti-Ro/SSA Abs determine the production of active TNF- $\alpha$ that stimulates SGEC to secrete the proinflammatory cytokines IL-6/IL- $8{ }^{8}$ TNF- $\alpha$ exerts some of its effects through activation of the proinflammatory transcription factor NF- $\kappa \mathrm{B} .{ }^{14}$ Recent studies were designed to explore the association of several inflammatory conditions with changes in TACE, TNF- $\alpha$ and NF- $\kappa$ B levels ${ }^{15,16}$ showing that pathways that include TNF- $\alpha$ signaling converge on NF- $\kappa \mathrm{B}$, contributing to exacerbate the diseases. NF- $\kappa \mathrm{B}$ is a dimeric transcription factor that is present in the cytosol in an inactive form bound to its inhibitory protein, inhibitory $\kappa \mathrm{B}$-alpha $(\mathrm{I} \kappa \mathrm{B}-\alpha)$. When activated by a variety of stimuli, $\mathrm{I} \kappa \mathrm{B}-\alpha$ is phosphorylated, ubiquitinated and is generally degraded in the proteosome releasing active NF- $\kappa \mathrm{B}$; this translocates into the nucleus and binds to a consensus site present within the promoter regions of genes such as those coding for proinflammatory cytokines. ${ }^{17}$

These findings prompted us to investigate into the relationship between TACE-mediated TNF- $\alpha$ release and the mechanisms by which anti-Ro/SSA Abs increase NF- $\kappa$ BDNA binding; proinflammatory genes induction was studied in an experimental model represented by human SGECs culture. The study was moreover designed to explore the involvement of TNF- $\alpha$ convertase TACE in the increased NF- $\kappa$ B-DNA binding through anti-Ro/SSA Abs-released cytokines propagating proinflammatory genes induction. As the feedback regulation of transcription factor NF- $\kappa \mathrm{B}$ by its inhibitor $\mathrm{I} \kappa \mathrm{B}-\alpha$ has an essential role in the control of NF- $\kappa \mathrm{B}$ activity, we investigated whether the TACE/TNF- $\alpha / N F-\kappa B$ pathway activated by anti-Ro/SSA Abs treatment is an I $\kappa \mathrm{B}-\alpha-$ dependent process.

\section{MATERIALS AND METHODS Autoantibodies}

Anti-Ro/SSA Abs were purified from IgG fractions of patients with primary SS ( $n, 33$; mean age 46.5 years), all fulfilling the American-European Consensus Group Classification criteria for SS. ${ }^{18}$ None of the patients examined met the clinical or serological criteria for additional connective tissue disease. None of the patients suffered from acute viral or bacterial infections at the time of the study. Informed consent for studies involving human subjects was obtained and the studies were conducted according to the tenets of the Declaration of Helsinki Principles. Anti-Ro/SSA Abs were purified from Sjögren IgG fractions ${ }^{19,20}$ using Sepharose 4B-Ro affinity columns. The affinity columns were prepared by coupling $5 \mathrm{mg}$ of Ro60 antigen (ImmunoVision, Springdale, AR, USA) per $1 \mathrm{ml}$ of CNBr-activated Sepharose 4B (Amersham Pharmacia Biotech, Sweden). Sjögren IgG was loaded and washed with 10 volumes of starting buffer. The anti-Ro/SSA Abs were eluted with $0.1 \mathrm{M}$ glycine (Sigma,
St. Louis, MO, USA), $\mathrm{pH} 2.5$, and neutralized immediately. The purity of the anti-Ro/SSA Abs was determined by SDSPAGE. The Abs obtained were concentrated to $200 \mu \mathrm{g} / \mathrm{ml}$ by the ultrafiltration method and used at concentrations of $50 \mu \mathrm{g} / \mathrm{ml}$ in the experimental procedures. Autoantibodies preparations resulted free of endotoxin contamination, as assessed by a Limulus amebocyte assay (Sigma). IgG from healthy subjects ( $\mathrm{HIgG})$ were obtained from control asymptomatic volunteers $(n, 15$; mean age 44.7 years).

\section{Microdissection and Primary Explant Culture}

Cultures of human healthy SGECs were derived from labial minor salivary gland (LSG) biopsy samples obtained from 10 healthy individuals awaiting removal of salivary mucoceles from the lower lip. Informed consent from the patients and approval by the local ethics committee was obtained. The healthy subjects had no complaints of oral dryness, no autoimmune disease and had normal salivary function. LSG samples were collected from the lower lip under local anesthesia through normal mucosa. The explant outgrowth technique $^{21}$ was applied to establish SGEC cultures from limited amounts of glandular tissue as previously reported. ${ }^{8}$

\section{Cell Treatment}

SGEC were collected by centrifugation at $250 \mathrm{~g}$ and resuspended at $1 \times 10^{6}$ cells $/ \mathrm{ml}$ in McCoy's 5a-modified medium. Cell suspensions $\left(10^{5}\right.$ cells/well $)$ were added to each well of a six-well plate (Thomas Scientific, Swedesboro, NJ, USA) and was allowed to incubate for $24 \mathrm{~h}$ at $37^{\circ} \mathrm{C}$ under $5 \%$ $\mathrm{CO}_{2}$. After incubation, cells were washed and treated as follows: (1) $\mathrm{IgG}$ fractions extracted from sera of healthy donors were dissolved in McCoy's $5 \mathrm{a}(50 \mu \mathrm{g} / \mathrm{ml})$ for $48 \mathrm{~h}$; (2) anti-Ro/SSA Abs $(50 \mu \mathrm{g} / \mathrm{ml})$ were dissolved in McCoy's $5 \mathrm{a}$ for $48 \mathrm{~h}$; and (3) exogenous TNF- $\alpha(500 \mathrm{pg} / \mathrm{ml})$ were dissolved in McCoy's $5 \mathrm{a}$ for $48 \mathrm{~h}$.

\section{Superarray Cytokine Genes Analysis}

Total cellular RNA was isolated by using Trizol (Qiagen, Valencia, CA, USA) and isopropanol precipitation or RNeasy mini kit (Qiagen) according to the manufacturer's instructions. Real-time PCR reactions for signaling pathway genes were analyzed in total RNA using the Human Inflammatory Response and Autoimmunity SuperArray $\mathrm{RT}^{2}$ Profiler (SABiosciences, Frederick, MD, USA) according to the manufacturer's protocol. The SuperArray combines SYBR Green-based real-time RT-qPCR technology with a multigene array plate format to simultaneously analyze 84 human cytokine and chemokines genes related to human inflammatory responses and autoimmunity. Briefly, cDNA was prepared from $1 \mu \mathrm{g}$ total RNA using a $\mathrm{RT}^{2}$ PCR array first-strand kit. The reaction was conducted in a $25 \mu \mathrm{l}$ mixture, which included Master mix, nuclease-free $\mathrm{H}_{2} \mathrm{O}$ and $1 \mu \mathrm{l}$ of template cDNA. PCR amplification was conducted with an initial $10 \mathrm{~min}$ step at $95^{\circ} \mathrm{C}$ followed by 40 cycles of $95^{\circ} \mathrm{C}$ for $15 \mathrm{~s}$ and $60^{\circ} \mathrm{C}$ for $1 \mathrm{~min}$. Real-time quantifications were performed 
using the ABI Prism 7700 fast real-time PCR system (Applied Biosystems, Foster City, CA, USA). The $\Delta \Delta C_{\mathrm{t}}$ method was used to analyze the expression level of each gene in comparison with five endogenous control housekeeping genes, present on the PCR array, that were used for normalization. Results were expressed as fold-changes in genes expression.

\section{Cytokine Proteins Array Analysis}

A human cytokine Multi-Analyte ELISArray Kit (SABiosciences) was used to estimate the cytokine production at the protein level, evaluating various cytokines (interleukin (IL)-1 $\alpha$, IL-1 $\beta$, IL-2, IL-4, IL-6, IL-8, IL-10, IL-12, IL-17A, interferon- $\gamma$ (IFN- $\gamma)$, TNF- $\alpha$ and granulocyte-macrophage colony-stimulating factor) in supernatants of SGEC treated with anti-Ro/SSA Abs or anti-Ro/SSA Abs plus TAPI-1. The arrays were performed according to the manufacturer's instructions. Levels of proteins were measured on a VERSAmax microplate reader (Molecular Devices, Silicon Valley, CA, USA) with an absorbance at $450 \mathrm{~nm}$. Sampling was performed in triplicates.

\section{Pharmacological Treatments to Inhibit TACE}

Human SGEC were pretreated with $10 \mu \mathrm{M}$ of the metalloprotease inhibitor TAPI-1 (Calbiochem, San Diego, CA, USA) or with the DMSO vehicle, used to dissolve the TAPI-1 for $1 \mathrm{~h}$, before stimulation with anti-Ro/SSA Abs or HIgG $(50 \mu \mathrm{g} / \mathrm{ml})$. This compound was neither cytotoxic at the concentration used nor did it interfere with the ability of SGEC to execute normal acinar and ductal morphogenesis in the presence of soluble EGF (data not shown).

\section{RT-PCR, Real-Time PCR, Western Blot and Flow Cytometry Analysis}

These procedures were conducted as described previously. ${ }^{8,13,19}$ For RT-PCR, the primers were designed according to published sequences (www.ncbi.nlm.nih.gov), and densitometric analysis was performed by gel image software (BIO-Profile Bio-1D; LTF Labortechnik GmbH, Wasserburg, Germany). For quantitative real-time PCR, sequences of the probes used are reported in Table 1.

\section{Immunofluorescence}

In the immunofluorescence assay, untreated and variously treated SGEC were incubated with the goat anti-human IL-13 $\mathrm{mAb}$, mouse anti-human IL-17 mAb and mouse anti-human IL-22 polyclonal $\mathrm{Ab}$ and rabbit anti-human NF- $\kappa \mathrm{B}$ p65 polyclonal Ab (all from Santa Cruz Biotechnology). The slides were incubated with related conjugated secondary antibodies (Santa Cruz Biotechnology) and examined using a confocal laser scanning microscopy system (Leica, TCS-SP2, Solms, Germany) using a $\lambda=488 \mathrm{~nm}$ Argon-Crypton laser for FITC.

\section{NF- $\kappa$ B DNA Binding Assays}

The ELISA-based TransAM Flexi NF- $\kappa \mathrm{B}$ p65 Kit (Active Motif, Carlsbad, CA, USA) was used to monitor the activity
Table 1 Primers and probes used in RT-PCR and real-time PCR assays

Sequences of the probes used in real-time PCR

\begin{tabular}{|c|c|}
\hline Target gene & Probe (5'-FAM and $3^{\prime}$-TAMRA labeled) \\
\hline TACE & $5^{\prime}$-CTGTGCAGTAGGACACGCCT-3' \\
\hline $\operatorname{LL}-1 \alpha$ & 5'-TCCACAGCGATTTATC-3' \\
\hline$I L-1 \beta$ & 5'-GGATTCAATGAGGAGACTTGC-3' \\
\hline IL-6 & 5'-CTGCCAAGAGAGCCACGGCCAG-3' \\
\hline IL-8 & 5'-TGCCCAAGAAGGCCACAGAACTGAA-3' \\
\hline IL-9 & 5'-TGCTGCCTCCAAGAACACAACTGA-3' \\
\hline IL-10 & $5^{\prime}$-CTTCCCTGTGAAAACAAGAGCAAGGCC-3' \\
\hline $1 L-13$ & 5'-TTGCCCAAGCAGGCCACAGAATTG-3' \\
\hline $1 L-17$ & 5'-CCTGGCCAAGGTCATCCATGACAACTT-3' \\
\hline $1 L-22$ & 5'-TCAGCCAGTTCCAGCTCCTCATGCA-3' \\
\hline$T N F-\alpha$ & 5'-TAGCCCATGTTGTAGCAAACCCTCAAGC-3' \\
\hline IL-10RA & 5'-CGAGGTGACCTGGGCACCATCC-3' \\
\hline IL-13RA & 5'-TAGCCCATGTTGTAGCAAACCCTCAA-3' \\
\hline
\end{tabular}

Abbreviations: IL, interleukin; TACE, TNF- $\alpha$ converting enzyme; TNF, tumor necrosis factor.

of NF- $\kappa \mathrm{B}$ p65 family members in the variously treated human SGEC. Nuclear fractions were collected from cells using the nuclear extraction kit following the manufacturer's suggestions (Active Motif) and the technique was applied as described previously. ${ }^{22}$

\section{Transient Transfection with the Dominant Negative Version of $I \kappa B-\alpha$ and Luciferase Assay}

To modulate NF- $\kappa$ B activity, healthy SGEC treated with antiRo/SSA Abs were transiently transfected with a dominant negative $\mathrm{I} \kappa \mathrm{B}-\alpha$ vector (the plasmid with the dominantnegative version of $\mathrm{I} \kappa \mathrm{B}-\alpha$ was a kind gift by Dr P Bauerle, University of Munich and Dr M Lienhard Schmitz, Deutsches Krebsforschungszentrum, Germany) or its control (empty vector, $\mathrm{pCMV}$ ). This dominant negative super-repressor I $\kappa \mathrm{B}-\alpha$ contains mutations at residues 32 and 36 (the two inducible phosphorylation sites), which disrupt phosphorylation and subsequent degradation. The super-repressor I $\kappa \mathrm{B}-\alpha$ mutant is still capable of binding NF- $\kappa \mathrm{B}$ and inhibiting DNA binding and NF- $\kappa$ B transcriptional activity. For transient transfections, $2 \times 10^{5}$ cells were seeded per well in a 24-well tissue culture dish one day before transient transfection. Cells were transfected with serum-free McCoy's 5a medium containing $25 \mu \mathrm{l} / \mathrm{ml}$ of Lipofectamine 2000 reagent (Invitrogen) and $10 \mu \mathrm{g} / \mathrm{ml}$ of dominant negative $\mathrm{I} \kappa \mathrm{B}-\alpha$ and a plasmid DNA including a NF- $\kappa$ B luciferase reporter construct or the empty vector (Clontech, San Diego, CA, USA), according to the manufacturer's instructions. This NF- $\kappa \mathrm{B}$ luciferase vector contains four tandem copies of the NF- $\kappa$ B 
consensus sequence fused to a TATA-like promoter region from the Herpes simplex virus thymidine kinase promoter. After $5 \mathrm{~h}$ of incubation, the medium was replaced with McCoy's 5a medium containing 10\% fetal bovine serum. Luciferase activity was measured by the Luciferase Reporter Assay System (Promega, Madison, WI, USA) according to the manufacturer's instructions. Luciferase activity was normalized to $\beta$-galactosidase activity as internal control. Each experiment was repeated at least three times.

\section{Statistics}

The data were analyzed for normality using the WilksShapiro test. Differences in means for paired observations were analyzed by the Student's $t$-test. In all instances, values of $P<0.05$ were considered statistically significant.

\section{RESULTS \\ TAPI-1 Inhibits the Expression of Inflammatory Cytokines/Chemokines in Anti-Ro/SSA Abs-Treated Human SGEC}

On the basis of the evidence that the cytokine network contributes to both systemic and exocrine manifestations of SS, it would be useful to know what effect this inflammatory process may have on the disease pathogenesis and whether an abnormal expression of these inflammatory molecules is able to alter the glandular integrity and functionality so significantly. Following the discovery of proinflammatory cytokines accumulation in functionally and structurally damaged areas of the salivary glands, ${ }^{3,6}$ and its pathogenic significance in autoimmune diseases, ${ }^{7,8}$ we assessed differences in the gene expression levels of inflammatory mediators, such as cytokines/chemokines, in human SGECs treated with the anti-Ro/SSA Abs that characterize SS. In anti-Ro/SSA Abs-treated SGEC we screened for abnormally expressed inflammatory mediators using $\mathrm{RT}^{2}$ Profiler PCR arrays, a high-throughput technique, designed specifically to target major cytokines and chemokines. Of the 84 genes examined (gene list in Supplementary Table), 27 genes were measurable in all the samples analyzed (Figure 1a); the other genes were either undetectable or else no technically satisfactory amplification was achieved. Anti-Ro/SSA Abs-treated SGEC exhibited differences as compared with untreated control cells and cells treated with HIgG. Anti-Ro/SSA treatment enhanced the expression of most of the measurable genes. Those increased by anti-Ro/SSA treatment include, among others, some of the CXC chemokines (CXCL1, CXCL6 and CXCL9), CC chemokines (CCL2, CCL13), interleukins (IL- $1 \alpha$, IL-1 $\beta$, IL-1F8, IL-6, IL-8, IL-9, IL-13, IL-17 and IL-22) and their receptors (IL-1RN, IL-10R $\alpha$, IL-13R $\alpha$, CCR1, CCR2, CCR3, CCR4 and CXCR1). The results demonstrated the presence of a pronounced inflammatory cell component in anti-Ro/SSAtreated SGEC that could contribute to the amplification of the epithelial-derived cytokine cascade.

As we have already demonstrated that anti-Ro/SSA Abs induced SGEC to express high levels of proinflammatory cytokines through a pathway involving $\mathrm{TACE}^{8}$ and the inhibitor TAPI-1 is known to prevent spontaneous membrane ectodomain shedding, including that of TNF- $\alpha,{ }^{23}$ we sought to investigate the possibility that TAPI-1 regulates proinflammatory cytokines production in anti-Ro/SSA Abs-treated SGEC. First we examined the efficacy of TAPI-1 on intracellular active TACE expression in anti-Ro/SSA Abstreated SGEC (Supplementary Figures S1, S2). As shown in Supplementary Figures, in anti-Ro/SSA Abs-treated SGEC, a clear reduction of TACE gene (Supplementary Figures S1a-c) and protein expression (Supplementary Figures S2a-c) was observed following TAPI-1 addition, confirming that TAPI-1 blocks the shedding of TACE in our experimental model. Once the effectiveness of TAPI- 1 is esatblished, we added this inhibitor to anti-Ro/SSA-treated SGEC to block TNF- $\alpha$ shedding through TACE and study the involvement of TACE/ TNF- $\alpha$ in proinflammatory cytokines secretion by anti-Ro/ SSA Abs-treated SGEC. TAPI-1-treated SGEC exhibited striking differences from anti-Ro/SSA Abs-treated cells and all genes examined were decreased by TAPI-1 (Figure 1a). To validate these findings, quantitative PCR was performed on RNA samples from an independent experiment using different primers from those on the array, which confirmed that TAPI-1 decreased the expression of the genes tested (Figure 1b). To estimate the cytokine production at protein level, a human cytokine ELISA array was utilized to evaluate various cytokines (IL- $1 \alpha$, IL- $1 \beta$, IL-2, IL-4, IL-6, IL-8, IL-10, IL-12, IL-17A, IFN- $\gamma$ and TNF- $\alpha$ ) in supernatants of SGEC treated with anti-Ro/SSA Abs or anti-Ro/SSA Abs plus TAPI-1. Among the cytokines that were consistently induced in SGEC by anti-Ro/SSA Abs treatment, the levels of all inflammatory cytokines were decreased in the supernatants of SGEC treated with anti-Ro/SSA Abs plus TAPI-1, showing statistically significant differences $(P<0.01)$ as compared with SGEC treated with anti-Ro/SSA Abs alone (Figure 1c). Thus, human SGEC cultures exposed to anti-Ro/SSA Abs plus TAPI-1 showed a decreased expression of proinflammatory cytokines mRNA and protein. Consistent with the role of TAPI-1 as TACE inhibitor in anti-Ro/SSA Absinduced-proinflammatory cytokines production, we performed an immunofluorescence assay demonstrating that TAPI-1 treatment resulted in a decreased production of the proinflammatory cytokines tested, such as IL-13, IL-17 and IL-22, in SGEC stimulated with anti-Ro/SSA Abs (Figure 1d). Given the widespread nature of cytokines expression changes induced by the TACE inhibitor TAPI-1, we postulated that TACE may regulate one or more genes that are central nodes in the inflammatory process linked to the autoimmune condition.

\section{Anti-Ro/SSA Abs Increased NF- $\kappa$ B-DNA Binding and NF- $\kappa$ B Target Gene Expression}

$\mathrm{NF}-\kappa \mathrm{B}$ is activated by various intracellular signals, including cytokines such as TNF- $\alpha$, and is a transcription factor for TNF- $\alpha$, which, in turn, induces NF- $\kappa$ B activation in a 
a

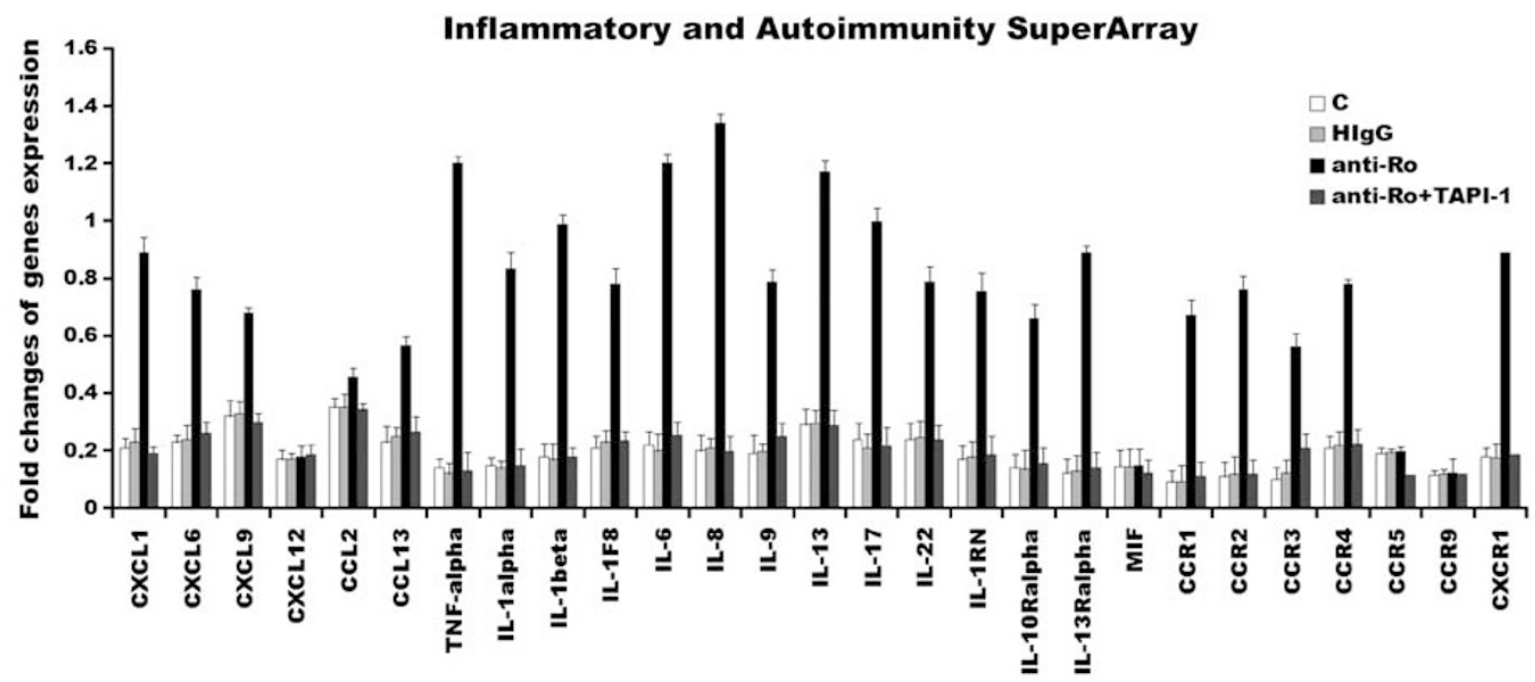

b

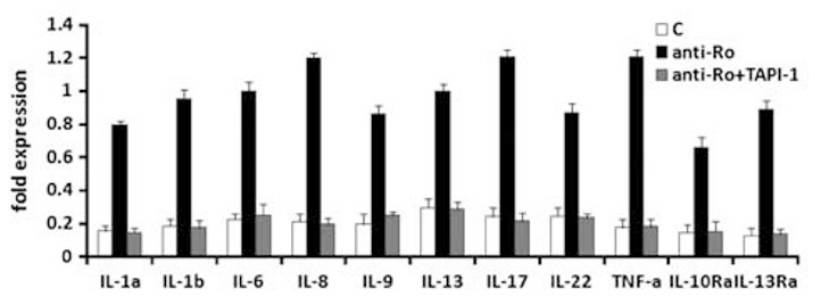

C

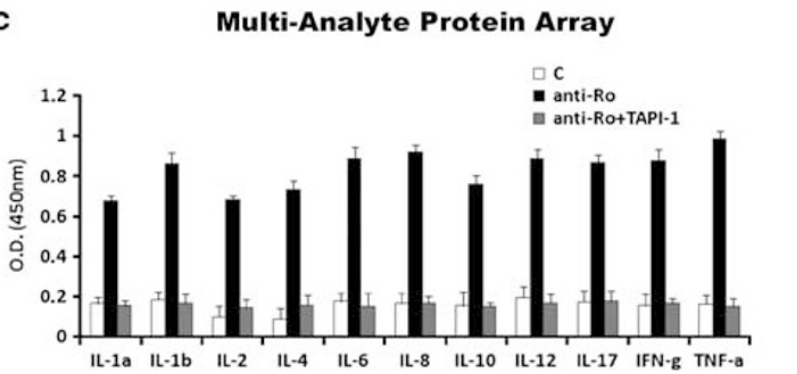

d

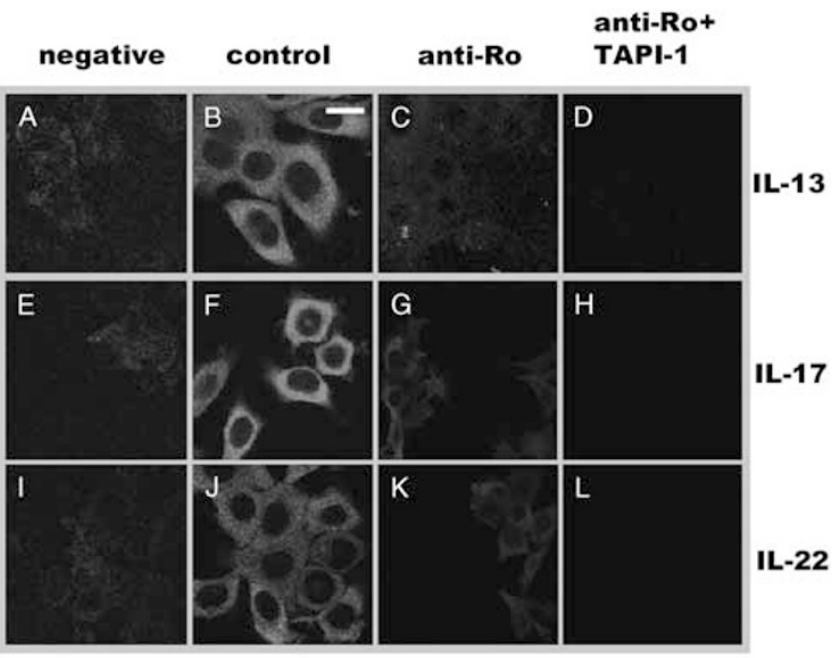

Figure 1 Blockade of TNF- $\alpha$ converting enzyme (TACE) activation decreased the anti-Ro/SSA autoantibodies (Abs)-induced expression of inflammationrelated genes. To demonstrate the differential regulation of proinflammatory cytokines/chemokines and receptors gene expression in anti-Ro/SSA Abs plus TAPI-1-treated human salivary gland epithelial cells (SGECs), an inflammatory response and autoimmunity SuperArray was adopted. Panel a shows that anti-Ro/SSA Abs treatment determines the differential expression of genes in human SGECs in comparison with untreated control cells.

The pooled total RNAs isolated from untreated and anti-Ro/SSA Abs-treated SGEC were used and real-time PCR arrays were run twice. From the expression of 84 key genes related to autoimmune and inflammatory response, 23 differentially expressed genes were identified as indicated. (Data represent the mean \pm s.e. of five independent experiments). Blockade of TACE activation with TAPI-1 reduces anti-Ro/SSA Abs-proinflammatory cytokines/chemokines induction. (b) Quantitative PCR validation of findings from superarray in panel a using primers and RNA samples from an independent experiment. The values represent the means of replicate samples and are normalized to housekeeping genes. (c) A human cytokine multi-analyte ELISA array was used to measure cytokine production by human SGEC treated with anti-Ro/SSA Abs plus TAPI-1. Values represent the optical density decrease versus anti-Ro/SSA Abs-treated SGEC cultures from triplicate wells. (Data represent the mean \pm s.e. of three independent experiments). (d) Intracellular distribution of interleukin (IL)-13, IL-17 and IL-22 in human SGECs treated with anti-Ro/SSA and/or TAPI-1 examined under fluorescence condition. A reduced immunoreactivity for cytokines expression was observed following TAPI-1 treatment (panels $C, G, K$ ). No fluorescence was detected in permeabilized cells treated only with secondary antibody-FITC (A, E, I).

positive feedback loop. ${ }^{24}$ As TNF- $\alpha$ is activated on the cell surface by TACE, we explored the pathway involving TACE/ TNF- $\alpha / \mathrm{NF}-\kappa \mathrm{B}$ in our experimental model of anti-Ro/SSA Abs-treated human SGEC. We previously reported that the transcription activity of NF- $\kappa \mathrm{B}$ is higher in SS than in normal subjects and human SGECs have a prominent role in orchestrating the salivary gland inflammatory response, suggesting that the NF- $\kappa \mathrm{B}$ signal is important in these cells for modulating immune responses. ${ }^{22} \mathrm{NF}-\kappa \mathrm{B}$ is a transcription factor family with multiple subunits, and the NF- $\kappa \mathrm{B}$ subunits p50 and p65 are commonly associated with increased gene transcription. Here we found that anti-Ro/SSA Abs 
increased the NF- $\kappa \mathrm{B}$ p65 nuclear immunofluorescence (Figures $2 \mathrm{a}$ and $\mathrm{c}$ ), whereas ELISA used to detect NF- $\kappa \mathrm{B}$ p65 subunits bound to the consensus DNA site of the identified region, demonstrated 2.65-fold more DNA binding by $\mathrm{p} 65$ in the nuclear extracts derived from anti-Ro/SSA Abs-treated SGEC as compared with untreated control SGEC (Figure 2b), suggesting that $\mathrm{p} 65$ transcription activity is higher after antiRo/SSA Abs treatment.

Proinflammatory cytokines are synthesized as larger protein precursors processed by proteases to become active mature forms. TNF- $\alpha$ is a prototype proinflammatory cytokine, and TACE is a prototype protease. ${ }^{25}$ Anti-Ro/SSA Abs treatment of SGEC cultures increased proinflammatory cytokines production and TACE mRNA and protein levels, suggesting that in human healthy SGEC, proinflammatory cytokines and proteases are induced during Abs exposure. ${ }^{8}$ As the induction of proinflammatory genes by anti-Ro/SSA Abs is complex and involves increased NF- $\kappa \mathrm{B}-\mathrm{DNA}$ binding, we tested the effect of TAPI- 1 on NF- $\kappa \mathrm{B}$ activation and NF- $\kappa$ B-mediated regulation of proinflammatory cytokines production. To test whether the TAPI-1 treatment concomitantly induced a decreased NF- $\kappa$ B activity, we evaluated the NF- $\kappa \mathrm{B}$ activity in anti-Ro/SSA Abs plus TAPI-1-treated SGEC, using the NF- $\kappa \mathrm{B}$ consensus DNA binding ELISA assay to detect bound NF- $\kappa \mathrm{B}$ p65 subunits. Our findings demonstrate changes in the levels of NF- $\kappa \mathrm{B}$ activation showing a clear reduction of NF- $\kappa$ B activity following TAPI-1 treatment (Figure 3). At the same time, to explore the association of anti-Ro/SSA Abs with changes in TACE, TNF- $\alpha$ and NF- $\kappa$ B, and demonstrate that the NF- $\kappa \mathrm{B}$ activation occurs through TACE, we inhibited TACE with TAPI-1 in anti-Ro/SSA Abstreated SGEC and, subsequently added exogenous soluble TNF- $\alpha$ to evaluate the NF- $\kappa$ B activation level (Figure 3). Analysis of the nuclear extracts revealed that after stimulation with exogenous soluble TNF- $\alpha$, in anti-Ro/SSA Abs plus TAPI-1-treated cells, p65 is observed to translocate into the nucleus, demonstrating that the specific TACE inhibitor blocks NF- $\kappa \mathrm{B}$ activation and nuclear translocation preventing release of the soluble form of TNF- $\alpha$.

\section{NF- $\kappa$ B Blockade by Dominant Negative I $\kappa$ B- $\alpha$ Decreased Proinflammatory Gene Expression}

Transcription is a complicated process involving many steps, out of which only one binds with DNA. To further investigate the role of NF- $\kappa \mathrm{B}$ on anti-Ro/SSA Abs-determined proinflammatory gene expression, we used the dominant negative super-repressor inhibitory protein $\mathrm{I} \kappa \mathrm{B}-\alpha$ as an inhibitor of NF- $\kappa \mathrm{B}-\mathrm{DNA}$ binding. NF- $\kappa \mathrm{B}$ is maintained in an inactive state in the cytoplasm bound to $\mathrm{I} \kappa \mathrm{B}-\alpha$, which masks its nuclear localization domain. ${ }^{1}$ Phosphorylation marks $\mathrm{I} \kappa \mathrm{B}-\alpha$ for degradation by the proteasome, causing NF- $\kappa \mathrm{B}$ nuclear translocation and activation. To evaluate the specific

a

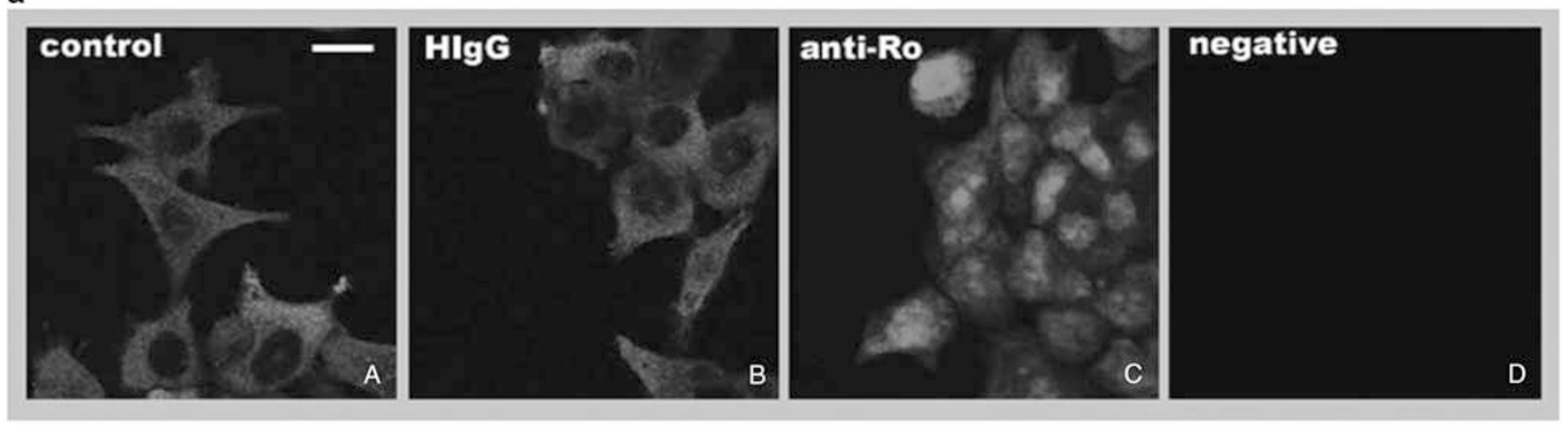

b

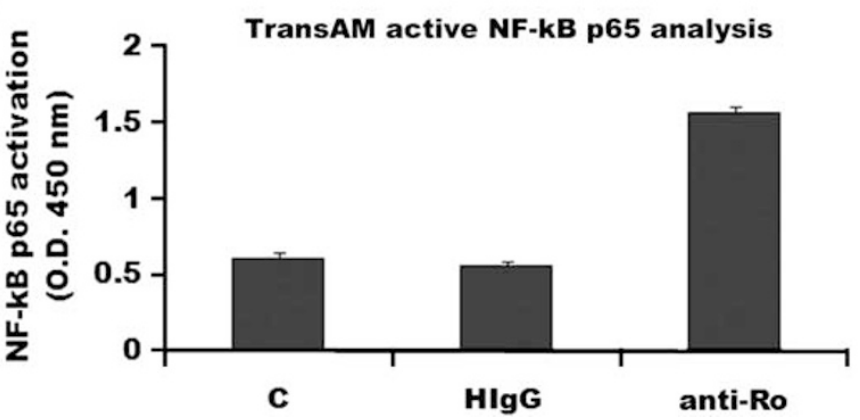

Figure 2 Anti-Ro/SSA autoantibodies (Abs) modulate nuclear factor- $\kappa \mathrm{B}$ (NF- $\kappa \mathrm{B}$ )-dependent transcriptional activity. (a) Confocal microscopy images from a representative experiment show anti-Ro/SSA Abs exposure increased NF- $\kappa$ B p65 nuclear immunofluorescence, as indicated in panel $C$. (b) Levels of NF- $\kappa$ B-motif-binding activity after $48 \mathrm{~h}$ of autoantibodies (Abs) stimulation. Nuclear extracts were isolated, and the level of endogenous NF- $\kappa \mathrm{B}$ (p65) transcriptional activation was examined by using a TransAM NF- $\kappa$ B kit. Values shown are the means \pm s.e. from six independent experiments. ELISA-based assay shows NF- $\kappa$ B subunit p65-DNA binding was increased following anti-Ro/SSA Abs exposure. 


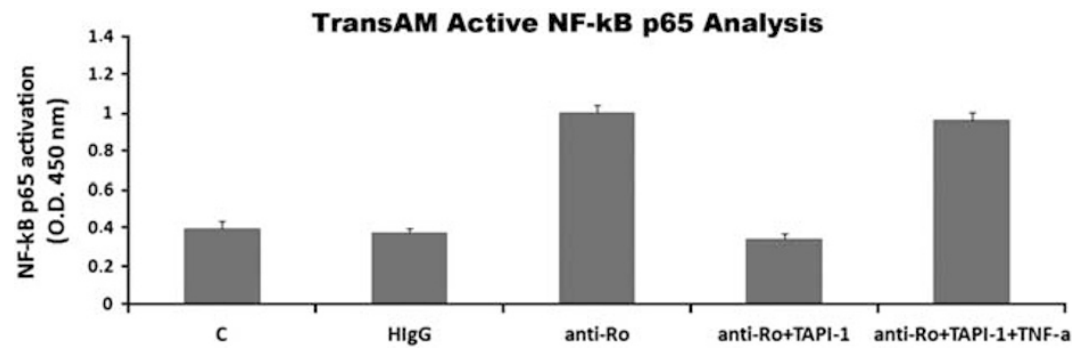

Figure 3 Tumor necrosis factor (TNF)- $\alpha$ converting enzyme (TACE) is involved in anti-Ro/SSA Abs-dependent nuclear factor- $\kappa B$ (NF- $\kappa B$ ) nuclear translocation. NF- $\kappa$ B activity evaluation in anti-Ro/SSA Abs plus TAPI-1-treated salivary gland epithelial cells (SGEC) using the NF- $\kappa$ B consensus DNA binding ELISA assay to detect bound NF- $\kappa$ B p65 subunits. The figure clearly demonstrates changes in the levels of NF- $\kappa$ B activation showing a reduction of NF- $\kappa$ B activity following TAPI-1 treatment. Analysis of the nuclear extracts revealed that, after stimulation with exogenous soluble TNF- $\alpha$, in anti-Ro/SSA Abs plus TAPI-1 treated cells, p65 is observed to translocate into the nucleus, demonstrating that the specific inhibitor of TACE blocks NF- $\kappa$ B activation and nuclear translocation preventing release of soluble form of TNF- $\alpha$. Experiments were repeated four times with similar design and results.

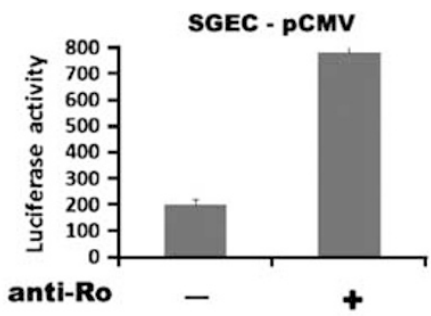

a

\section{b}

Inflammatory and Autoimmunity SuperArray

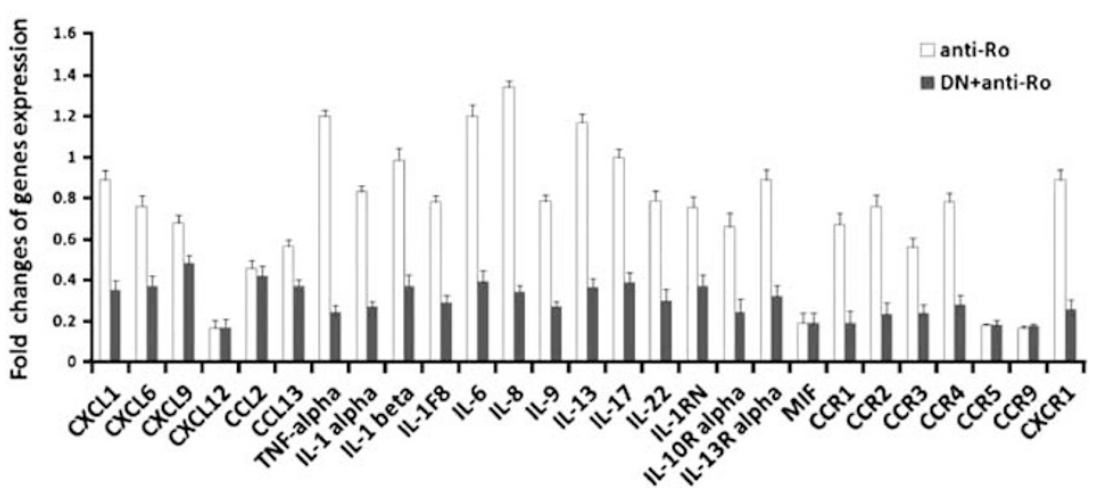

Figure 4 Blockade of nuclear factor- $\kappa \mathrm{B}$ (NF- $\kappa$ B) activation through inhibitory $\kappa \mathrm{B}$-alpha $(\mid \kappa \mathrm{B}-\alpha)$ dominant negative blunts anti-Ro/SSA autoantibodies (Abs) induction of proinflammatory genes. (a) Effect of dominant negative super-repressor $\mid \kappa \mathrm{B}-\alpha(\mathrm{I} \kappa \mathrm{B}-\alpha \mathrm{DN})$ transfection on NF- $\kappa \mathrm{B}$ activation in anti-Ro/SSA Abs-treated salivary gland epithelial cells (SGECs). Healthy SGECs were transiently transfected with serum-free McCoy's $5 a$ medium containing $25 \mu \mathrm{l} / \mathrm{ml}$ of Lipofectamine 2000 reagent and $10 \mu \mathrm{g} / \mathrm{ml}$ of $I_{\kappa} \mathrm{B}-\alpha \mathrm{DN}$ (that contains mutations at residues 32 and 36 inducible phosphorylation sites) and a plasmid DNA including a NF- $\kappa$ B luciferase reporter construct or the empty vector (pCMV) according to the manufacturer's instructions. At $48 \mathrm{~h}$ after transfection, cells were treated with anti-Ro/SSA Abs and cell extracts were prepared for luciferase activity assay as described in Materials and Methods. Results were normalized to $\beta$-galactosidase activity and plotted as the means of triplicates of a representative experiment out of at least three independent experiments. (b) Blokade of NF- $\kappa$ B through the super-repressor $I \kappa B-\alpha$ DN affect inflammation-related genes expression. Human SGEC were screened with real-time PCR array for changes in the expression of inflammation-related genes after transfection with $1 \kappa \mathrm{B}-\alpha \mathrm{DN}$ and treatment with anti-Ro/SSA Abs. Removal of the $\mathrm{I} \kappa \mathrm{B}-\alpha$ feedback regulation of NF- $\kappa \mathrm{B}$ resulted in prolonged NF- $\kappa \mathrm{B}$ activation that alters anti-Ro/SSA-dependent proinflammatory cytokines expression. (Data represent the mean \pm s.e. of three independent experiments).

contribution of phosphorylation of $\mathrm{I} \kappa \mathrm{B}-\alpha$ at serine $32-36$ (the two inducible phosphorylation sites) on TACE-induced NF- $\kappa \mathrm{B}$ activation in anti-Ro/SSA Abs-treated healthy SGEC, we used a mutant form of $\mathrm{I} \kappa \mathrm{B}-\alpha$. This dominant negative super-repressor $\mathrm{I} \kappa \mathrm{B}-\alpha$ contains mutations at residues 32 and 36, which disrupt phosphorylation and subsequent degradation. The super-repressor $\mathrm{I} \kappa \mathrm{B}-\alpha$ mutant is still capable of binding NF- $\kappa \mathrm{B}$ and inhibiting DNA binding and NF- $\kappa \mathrm{B}$ transcriptional activity. The healthy SGEC were treated with anti-Ro/SSA Abs after transfection with a vector containing the mutated variant of $\mathrm{I} \kappa \mathrm{B}-\alpha$ and NF- $\kappa \mathrm{B}$ activation was determined by luciferase assay. Luciferase activity was evaluated by measuring photon emission after incubation with the luciferin substrate. As shown in Figure 4a, transfection of 
the dominant negative super-repressor $\mathrm{I} \kappa \mathrm{B}-\alpha$ significantly attenuated NF- $\kappa \mathrm{B}$ activity, resulting in a 1.6-fold reduction in photon emission. In direct contrast, anti-Ro/SSA Abs treatment of healthy SGEC that present the non-mutated form of $\mathrm{I} \kappa \mathrm{B}-\alpha(\mathrm{pCMV})$ caused a 5.5 -fold increase in reporter expression. To further examine whether targeting the NF- $\kappa \mathrm{B}$ pathway using the dominant negative super-repressor $\mathrm{I} \kappa \mathrm{B}-\alpha$ protein affects inflammatory mediators, cells were screened with a real-time PCR array for changes in the expression of inflammation-related genes. Transfection with dominant negative $\mathrm{I} \kappa \mathrm{B}-\alpha$ in anti-Ro/SSA-treated SGEC determined a marked reduction of proinflammatory cytokines gene expression (Figure 4b). This array analysis suggests that removal of the feedback regulation of NF- $\kappa \mathrm{B}$ by $\mathrm{I} \kappa \mathrm{B}-\alpha$ resulted in prolonging NF- $\kappa \mathrm{B}$ activation, which sequentially altered the expression of many NF- $\kappa \mathrm{B}$ target genes, such as proinflammatory cytokines. These studies further support an Abs-increased NF- $\kappa \mathrm{B}-\mathrm{DNA}$ binding with increased proinflammatory gene transcription.

\section{DISCUSSION}

NF- $\kappa \mathrm{B}$ regulates the transcription of innate proinflammatory immune genes as well as many other genes. ${ }^{26}$ Most cell types obtained from patients with autoimmune diseases, unlike normal cells, constitutively express active NF- $\kappa \mathrm{B}$, through a mechanism that is not fully understood. We report herein that anti-Ro/SSA Abs exposure of human SGECs progressively increases NF- $\kappa \mathrm{B}-\mathrm{DNA}$ binding and proinflammatory genes expression. The induction of proinflammatory genes by anti-Ro/SSA Abs is complex, involving increased NF- $\kappa \mathrm{B}-$ DNA binding, in part through anti-Ro/SSA Abs-released cytokines propagating proinflammatory gene induction, associated with the activation of the TNF- $\alpha$ convertase TACE. This finding supports a possible therapeutic role for specific TACE inhibitors in autoimmune diseases such as SS.

In previous studies, we investigated the pathogenic role of anti-Ro/SSA Abs, the anti-nuclear Abs characterizing SS and primarily directed against a number of antigenic proteins coupled to small RNA molecules. We demonstrated that Abs can access SGEC via $\mathrm{Fc} \gamma$ receptors, transmembrane receptors that recognize the $\mathrm{Fc}$ part of the IgG, leading to various functional cellular alterations. ${ }^{20,27}$ SGEC express the TNF- $\alpha$ receptors and produce the TNF- $\alpha$ cytokine following anti-Ro/SSA Abs internalization ${ }^{28}$ responsible for the constitutive activation of NF- $\kappa \mathrm{B}$ observed in SGECs derived from patients with SS. The active NF- $\kappa$ B was associated with the downregulation of TNFAIP3, that is, a negative feedback regulator of TNF- $\alpha$ signaling through NF- $\kappa B .{ }^{24}$ TNF- $\alpha$ is a prototype target gene of the NF- $\kappa \mathrm{B}$ signaling pathway and may function in an autocrine/paracrine manner to propagate cytokine production and activate the NF- $\kappa \mathrm{B}$ signaling pathway. ${ }^{29,30}$ Furthermore, we previously found that both TNF- $\alpha$ and TACE were induced by anti-Ro/SSA Abs treatment of human SGECs cultures, and the TACE-TNF- $\alpha$ amphiregulin axis could be involved in one of the mechanism responsible for the proinflammatory cytokines overexpression characterizing SS. ${ }^{8}$

In this context, anti-Ro/SSA Abs induction of NF- $\kappa \mathrm{B}-$ DNA binding and target proinflammatory gene expression were studied in this work using the TACE inhibitor TAPI-1 that has been validated in preclinical trials for the treatment of autoimmune diseases. ${ }^{31}$ In addition, to evaluate the specific contribution of $\mathrm{I} \kappa \mathrm{B}-\alpha$ phosphorylation to TACEinduced NF- $\kappa \mathrm{B}$ activation in SGEC, we used the dominant negative super-repressor $\mathrm{I} \kappa \mathrm{B}-\alpha$, which disrupts phosphorylation and subsequent degradation, preventing NF- $\kappa \mathrm{B}$ nuclear translocation. Following the discovery of proinflammatory cytokines accumulation in functionally and structurally damaged areas of the salivary glands, ${ }^{3,6}$ we first assessed any differences in the level of gene expression of inflammatory mediators, such as cytokines/chemokines, in human SGECs treated with the anti-Ro/SSA Abs characterizing SS. The screening for abnormally expressed inflammatory mediators demonstrated that anti-Ro/SSA Abs enhanced the expression of CXC chemokines, CC chemokines, several ILs and their receptors. To confirm results obtained in healthy SGEC under anti-Ro/SSA Abs treatment, we evaluated the expression of some selected cytokines (IL-13, IL-17, IL-22) in the SS labial salivary glands biopsy samples, demonstrating a strong positive staining for proinflammatory cytokines located in the cytoplasm of acini and ductal cells. The combined study conducted on SS biopsy samples, and anti-Ro/SSA Abs-treated SGEC supports the presence of a pronounced inflammatory cell component in SS and demonstrated that anti-Ro/SSA Abs contribute to the amplification of the epithelial-derived cytokine cascade. It has been hypothesized that small-sized, orally bioavailable TACE inhibitors might have the potential to effectively treat inflammatory diseases by limiting the levels of soluble TNF- $\alpha$, that has an important role in inflammatory disorders of both inflammatory and non-inflammatory origin. ${ }^{32}$ It has also been demonstrated that inhibition of TACE by small-molecular-weight orally bioavailable drugs is effective in blocking downstream cytokine production. ${ }^{33}$ On the basis of this hypothesis, we sought to investigate the possibility that TAPI-1 regulates proinflammatory cytokines production in anti-Ro/SSA Abs-treated SGEC. We found that the addition of TAPI-1 to anti-Ro/SSA Abs-treated SGEC reduced the inflammation and TAPI-1 led to a marked reduction of proinflammatory cytokines production. A human cytokine array was utilized to evaluate various cytokines (IL- $1 \alpha$, IL- $1 \beta$, IL-2, IL-4, IL-6, IL-8, IL-10, IL-12, IL-17A, IFN- $\gamma$, TNF- $\alpha$ ) at messenger RNA and protein level. All inflammatory cytokines were decreased in the supernatants of SGEC treated with anti-Ro/SSA Abs plus TAPI-1, demonstrating that TACE inhibition may be an attractive approach consisting of interfering with circulating levels of TNF- $\alpha$ to treat inflammatory autoimmune diseases.

Recently, substantial progress has been made in understanding the mechanisms that control the dynamics of NF- $\kappa$ B 
activation and several studies are attempting to clarify the effect of activation of the TACE/TNF- $\alpha / N F-\kappa$ B pathway in inflammatory states. ${ }^{16}$ In this study, we explored the putative role of TACE on NF- $\kappa \mathrm{B}$ activation in an autoimmune disorder. NF- $\kappa$ B has been shown to be chronically active in many inflammatory autoimmune diseases such as inflammatory bowel disease, ${ }^{34}$ rheumatoid arthritis ${ }^{35}$ and systemic lupus erythematosus ${ }^{36}$ as well as SS. ${ }^{22}$ In fact, we previously demonstrated enhanced levels of NF- $\kappa$ B activation in SS as compared with normal subjects, suggesting that the NF- $\kappa \mathrm{B}$ signal is important in human salivary gland cells for modulating immune responses. ${ }^{22}$ In the present study we found that SS Abs are able to trigger the signal transduction pathways involved in NF- $\kappa \mathrm{B}$ activation leading to enhanced gene expression of proinflammatory mediators. As the induction of proinflammatory genes by anti-Ro/SSA Abs is complex and involves increased NF- $\kappa \mathrm{B}-\mathrm{DNA}$ binding, we tested the effect of TAPI- 1 on NF- $\kappa$ B activation and NF- $\kappa$ B-mediated regulation of proinflammatory cytokines production. We found that administration of the TACE inhibitor TAPI-1 blocks the anti-Ro/SSA Abs-stimulated translocation of NF- $\kappa \mathrm{B}$ to the nucleus and this occurs through TACE. This was confirmed by the addition of exogenous soluble TNF- $\alpha$ to anti-Ro/SSA Abs-treated SGEC in which we neutralized TACE with TAPI-1. After stimulation with exogenous soluble TNF- $\alpha, \mathrm{NF}-\kappa \mathrm{B}$ is observed to translocate to the nucleus, demonstrating that TAPI-1 blocks NF- $\kappa \mathrm{B}$ activation preventing the release of the soluble form of TNF- $\alpha$.

The next step was to study the role of NF- $\kappa \mathrm{B}$ on anti-Ro/ SSA Abs-determined proinflammatory gene expression in depth, using the dominant negative super-repressor inhibitory protein $\mathrm{I} \kappa \mathrm{B}-\alpha . \mathrm{I} \kappa \mathrm{B}-\alpha$ is an essential regulator of the $\mathrm{NF}-\kappa \mathrm{B}$ transcription factor, which orchestrates the expression of a plethora of genes that are essential for controlling apoptosis, cell proliferation, and immune and inflammatory responses. ${ }^{37,38}$ One of the key target genes induced by NF- $\kappa \mathrm{B}$ is its inhibitor $\mathrm{I} \kappa \mathrm{B}-\alpha$, which in turn inhibits NF- $\kappa \mathrm{B}$ activity and thus establishes a feedback regulation mechanism for controlling NF- $\kappa \mathrm{B}$ activity. ${ }^{39}$ Our findings demonstrate a central role of the feedback regulation of NF- $\kappa \mathrm{B}$ activity by $\mathrm{I} \kappa \mathrm{B}-\alpha$ in the control of proinflammatory cytokines production induced by SS Abs. Our real-time PCR array results suggest that removal of the feedback regulation of NF- $\kappa \mathrm{B}$ by $\mathrm{I} \kappa \mathrm{B}-\alpha$ resulted in prolonging NF- $\kappa \mathrm{B}$ activation, which sequentially altered the expression of proinflammatory cytokines.

Taken together, these studies are consistent with the hypothesis that immune activation and inflammatory cytokines such as TNF- $\alpha$ have an important role in the origin and progression of SS. As TACE is helpful in processing many substrates, its inhibition might be successfully used in diseases in which TNF- $\alpha$ antagonism has been validated. Although further studies are needed to clarify the mechanisms underlying SS, our results demonstrate that SS Abs exert their pathogenic effects via triggering the TACE/TNF- $\alpha /$
NF- $\kappa \mathrm{B}$ axis. Furthermore, our findings revealed an essential role of the feedback regulation of NF- $\kappa \mathrm{B}$ activity by $\mathrm{I} \kappa \mathrm{B}-\alpha$ in monitoring the proinflammatory cytokines production observed in SS, suggesting that NF- $\kappa \mathrm{B}$ signaling may be a potential therapeutic target in SS and other autoimmune diseases. The novel mechanism described in our manuscript could offer potential targets for the treatment of pathologies associated with increased TNF- $\alpha$ levels and new therapeutic developments such as agents that modulate the production of TNF- $\alpha$. The benefit of such targeted therapy will depend on the delicate balance between suppressing inflammation and interfering with normal cellular functions. By selectively targeting TACE or specific NF- $\kappa \mathrm{B}$ subunits or $\mathrm{I} \kappa \mathrm{B}-\alpha$ proteins that have a degree of tissue specificity, one might attain therapeutic efficacy and minimize systemic toxicity.

Supplementary Information accompanies the paper on the Laboratory Investigation website (http://www.laboratoryinvestigation.org)

\section{ACKNOWLEDGEMENTS}

We are grateful to Dr P Bauerle, University of Munich and to Dr M Lienhard Schmitz, Deutsches Krebsforschungszentrum, Germany, for kindly giving us the plasmid with the dominant negative version of $\mid \kappa \mathrm{B}-\alpha$. This work was supported by Grant (No: 20216000056) from the Italian Ministry for Universities and Research. We thank MVC Pragnell, BA, for the critical reading of the manuscript.

\section{DISCLOSURE/CONFLICT OF INTEREST}

The authors declare no conflict of interest.

1. Hayden MS, Ghosh S. Shared principles in NF-kappaB signaling. Cell 2008;132:344-362.

2. Brown KD, Claudio E, Siebenlist U. The roles of the classical and alternative nuclear factor-kappaB pathways: potential implications for autoimmunity and rheumatoid arthritis. Arthritis Res Ther 2008;10:212.

3. Roescher N, Tak PP, Illei GG. Cytokines in Sjögren's syndrome. Oral Dis 2009;15:519-526.

4. Fox $\mathrm{Rl}$, Kang $\mathrm{HI}$, Ando $\mathrm{D}$, et al. Cytokine mRNA expression in salivary gland biopsies of Sjogren's syndrome. J Immunol 1994;152:5532-5539.

5. Nikolov NP, Illei GG. Pathogenesis of Sjögren's syndrome. Curr Opin Rheumatol 2009;21:465-470.

6. Cuello $C$, Palladinetti $P$, Tedla $N$, et al. Chemokine expression and leucocyte infiltration in Sjögren's syndrome. Br J Rheumatol 1998;37: 779-783.

7. Sisto $\mathrm{M}$, Lisi $\mathrm{S}$, Lofrumento $\mathrm{DD}$, et al. Expression of pro-inflammatory TACE-TNF- $\alpha$-amphiregulin axis in Sjögren's syndrome salivary glands. Histochem Cell Biol 2010;134:345-353.

8. Lisi S, Sisto M, Lofrumento DD, et al. Pro-inflammatory role of anti-Ro/ SSA autoantibodies through the activation of Furin-TACEamphiregulin axis. J Autoimmun 2010;35:160-170.

9. Ewert $P$, Aguilera $S$, Alliende $C$, et al. Disruption of tight junction structure in salivary glands from Sjögren's syndrome patients is linked to proinflammatory cytokine exposure. Arthritis Rheum 2010;62: 1280-1289.

10. Pflugfelder SC, Jones D, Ji Z, et al. Altered cytokine balance in the tear fluid and conjunctiva of patients with Sjögren's syndrome keratoconjunctivitis sicca. Curr Eye Res 1999;19:201-211.

11. Black RA, Rauch CT, Kozlosky CJ, et al. A metalloproteinase disintegrin that releases tumour-necrosis factor- $\alpha$ from cells. Nature 1997;385:729.

12. Moss $\mathrm{ML}$, Jin $\mathrm{SL}$, Becherer JD, et al. Cloning of a disintegrin metalloproteinase that processes precursor tumour-necrosis factor- $\alpha$. Nature 1997;385:733.

13. Sisto $M$, Lisi $S$, Lofrumento $\mathrm{DD}$, et al. TNF blocker drugs modulate human TNF- $\alpha$-converting enzyme pro-domain shedding induced by autoantibodies. Immunobiology 2010;215:874-883. 
14. Liu ZG. Molecular mechanism of TNF signaling and beyond. Cell Res 2005;15:24-27.

15. Madrigal $\mathrm{JL}$, Hurtado $\mathrm{O}$, Moro MA, et al. The increase in TNF-alpha levels is implicated in NF-kappaB activation and inducible nitric oxide synthase expression in brain cortex after immobilization stress. Neuropsychopharmacology 2002;26:155-163.

16. Bigdeli MR, Khoshbaten A. In vivo preconditioning with normobaric hyperoxia induces ischemic tolerance partly by triggering tumor necrosis factor-alpha converting enzyme/tumor necrosis factor-alpha/ nuclear factor-kappaB. Neuroscience 2008;153:671-678.

17. Zheng $C$, Yin $Q$, Wu $H$. Structural studies of NF- $\kappa$ B signaling. Cell Res 2011;21:183-195.

18. Vitali C, Bombardieri S, Jonsson R, et al. Classification criteria for Sjögren's syndrome: a revised version of the European criteria proposed by the American-European Consensus Group. European Study Group on Classification Criteria for Sjögren's Syndrome. Ann Rheum Dis 2002;61:554-558.

19. Sisto M, Lisi S, Castellana D, et al. Autoantibodies from Sjögren's syndrome induce activation of both the intrinsic and extrinsic apoptotic pathways in human salivary gland cell line A-253. J Autoimmun 2006;27:38-49.

20. Lisi S, Sisto $M$, Soleti $R$, et al. Fcgamma receptors mediate internalization of anti-Ro and anti-La autoantibodies from Sjögren's syndrome and apoptosis in human salivary gland cell line A-253. J Oral Pathol Med 2007;36:511-523.

21. Sens DA, Hintz DS, Rudisill MT, et al. Explant culture of human submandibular gland epithelial cells: evidence for ductal origin. Lab Invest 1985;52:559-567.

22. Sisto M, Lisi S, Lofrumento DD, et al. A failure of TNFAIP3 negative regulation maintains sustained NF- $\kappa B$ activation in Sjögren's syndrome. Histochem Cell Biol 2011;135:615-625.

23. Crowe PD, Walter BN, Mohler KM, et al. A metalloprotease inhibitor blocks shedding of the $80-\mathrm{kD}$ TNF receptor and TNF processing in T lymphocytes. J Exp Med 1995;181:1205-1210.

24. Coward WR, Okayama Y, Sagara H, et al. NF-kappa B and TNF-alpha: a positive autocrine loop in human lung mast cells? J Immunol 2002; 169:5287-5293.
25. Edwards DR, Handsley MM, Pennington CJ. The ADAM metalloproteinases. Mol Aspects Med 2008;29:258-289.

26. Perkins ND. Integrating cell-signalling pathways with NF-kappaB and IKK function. Nat Rev Mol Cell Biol 2007;8:49-62.

27. Lisi $S, D^{\prime}$ Amore $M$, Lofrumento $D$, et al. Modulation of the Fc gamma receptors induced by anti-Ro and anti-La autoantibodies: observations in salivary gland cells. Rheumatol Int 2008;28:943-948.

28. Sisto $M$, Lisi $S, D^{\prime}$ Amore $M$, et al. Anti-Ro and anti-La autoantibodies induce TNF-alpha production by human salivary gland cells: an in vitro study. Reumatismo 2007;59:221-226.

29. Hayakawa K, Meng Y, Hiramatsu N, et al. Spontaneous activation of the NF-kappaB signaling pathway in isolated normal glomeruli. Am J Physiol Renal Physiol 2006;291:F1169-F1176.

30. Syed MM, Phulwani NK, Kielian T. Tumor necrosis factor-alpha (TNF-alpha) regulates Toll-like receptor 2 (TLR2) expression in microglia. J Neurochem 2007;103:1461-1471.

31. Arribas J, Esselens C. ADAM17 as a therapeutic target in multiple diseases. Curr Pharm Des 2009;15:2319-2335.

32. Strieter RM, Kunkel SL, Bone RC. Role of tumor necrosis factor-a in disease states and inflammation. Crit Care Med 1993;21:S447-S463.

33. Newton $\mathrm{RC}$, Solomon $\mathrm{KA}$, Covington $\mathrm{MB}$, et al. Biology of TACE inhibition. Ann Rheum Dis 2001;60:iii25-iii32.

34. Atreya I, Atreya R, Neurath MF. NF-kappaB in inflammatory bowel disease. J Intern Med 2008;263:591-596.

35. Roman-Blas JA, Jimenez SA. NF-kappaB as a potential therapeutic target in osteoarthritis and rheumatoid arthritis. Osteoarthritis Cartilage 2006;14:839-848.

36. Valéro R, Baron ML, Guérin S, et al. A defective NF-kappa B/RelB pathway in autoimmune-prone New Zealand black mice is associated with inefficient expansion of thymocyte and dendritic cells. J Immunol 2002;169:185-192.

37. Li Q, Verma IM. NF-kappaB regulation in the immune system. Nat Rev Immunol 2002;2:725-734.

38. Ghosh S, Karin M. Missing pieces in the NF-kappaB puzzle. Cell 2002;109(Suppl):S81-S96.

39. Chiao PJ, Miyamoto S, Verma IM. Autoregulation of I kappa B alpha activity. Proc Natl Acad Sci USA 1994;91:28-32. 